\title{
Normal and shear forces in the contact patch of a braked racing tyre Part 1: Results from a Finite Element model
}

\author{
Patrick Gruber*, Robin S Sharp, and Andrew D Crocombe
}

Division of Mechanical, Medical, and Aerospace Engineering, Faculty of Engineering and Physical Sciences, University of Surrey, Guildford, United Kingdom

*Corresponding author: Division of Mechanical, Medical, and Aerospace Engineering (A1), Faculty of Engineering and Physical Sciences, University of Surrey, Guildford, GU2 7XH, UK. email: p.gruber@ surrey.ac.uk

This is a preprint of an article whose final and definitive form has been published in Vehicle System Dynamics (C 2012, copyright Taylor \& Francis; Vehicle System Dynamics is available online at: www.tandfonline.com/10.1080/00423114.2011.586428. 


\section{Normal and shear forces in the contact patch of a braked racing tyre Part 1: Results from a Finite Element model}

In this first part of a two-part article, a previously-described and validated finiteelement model of a racing-car tyre is developed further to yield detailed information on carcass deflections and contact pressure and shear stress distributions for a steadyrolling, slipping and cambered tyre. Variations in running conditions simulated include loads of 1500,3000 and $4500 \mathrm{~N}$, camber angles of $0^{\circ}$ and $-3^{\circ}$, and longitudinal slips from 0 to $-20 \%$. Special attention is paid to heavy braking, in which context the aligning moment is of great interest.

Results generated are in broad agreement with limited experimental results from the literature and they provide considerable insight into how the tyre deforms and how the contact stresses are distributed as functions of the running conditions. Generally, each rib of the tyre behaves differently from the others, especially when the wheel is cambered.

The results form a basis for the development of a simpler physical tyre model, the purpose of which is to retain accuracy over the full operating range while demanding much less computational resource. The physical tyre model is the topic of the second part of the article.

Keywords: finite element, steady-state rolling, rolling radius, shear forces, contact patch, racing tyre

\section{Introduction}

Modern radial tyres consist of several rubber compounds and reinforcement layers, forming a highly anisotropic composite structure. On a running car, this complex ply system generates a network of deformations which influence the distribution of the contact stresses at the tread-road interface and, thus, the force generation capability of the whole tyre. A better understanding of these contact patch characteristics is therefore key to enhancing knowledge about the rolling behaviour of tyres. In connection with racing, insights into the characteristics of the contact stress distribution are of particular interest as they are intimately connected with the controllability and, hence, the performance of the entire vehicle.

For this study, a racing tyre is examined under one of the most severe operating conditions - that is, heavy braking in a straight line. By making detailed observations of the deformations and stresses, a braking manoeuvre allows valuable insights into many details of a rolling tyre, ranging from complex interactions between structural characteristics and contact stresses to overall tyre force and moment characteristics. For this kind of investigation two main test methods are possible - experimental testing and virtual testing. In 
practice, if the experimental tests are performed, many problems arise, as local measurements on a rolling tyre are intricate and difficult. With virtual testing, the experimental difficulties are avoided, but they are replaced by the need for a model that accurately replicates the behaviour of the real tyre. Using the finite element (FE) method, the construction of such a model was the subject of previous research. The created and validated full three-dimensional FE model is described in detail in [1]. In line with current research in the field of tyre simulations (e.g., $[2,3])$, this virtual tyre is developed further for the work here, which involves numerical tests of a braked tyre at different vertical loads, wheel camber angles and longitudinal slip ratios. In contrast to the work of earlier researchers, the obtained results are analysed and presented in novel ways, yielding new insights into tyre behaviour. Also, numerical limitations of virtual testing of a rolling tyre are discussed.

Insights from this study form a basis for the development of a simpler physical tyre model that retains accuracy over the full operating range while reducing computational demand. The physical tyre model is the topic of the second part of the article.

\section{Model setup}

This investigation makes use of the previously-described and validated full three-dimensional FE model of a racing tyre (Figure 1). Results from the previous research on static stiffness properties demonstrate that the virtual tyre captures real tyre behaviour in great detail. Close examination of the contact patch allowed identification of complex interactions between the deflection characteristics of the carcass and the shear stress distribution. In particular, at low friction levels the racing tyre exhibits lateral sliding at the tread-road interface which leads to "waisting" of the contact region.

As will be discussed below, the previously-described FE model is further developed to allow simulation of steady-state rolling motion. All simulations are conducted with the implicit commercial code Abaqus/Standard V6.7 (Dassault Systèmes, Paris, France). Within 
the following discussion, the right-hand orthogonal ISO axis system is employed [4]. The origin of this system is in the centre of the contact patch, which is defined as the intersection of the wheel centre plane with the vertical projection of the wheel spin axis onto the road plane. The $\mathrm{x}$-axis is the intersection of the wheel centre plane with the road plane with a positive direction taken for the tyre moving forward. The z-axis is perpendicular to the road surface and points upwards. The y-axis is determined by the right-hand rule.

\subsection{Overview of virtual testing}

The rolling motion of the tyre is simulated with the 'Steady-State-Transport' (SST) capability of Abaqus, which employs a mixed Lagrangian-Eulerian simulation technique [5]. With this method, the reference frame for the mesh is fixed and the movement of the tyre is simulated by transporting the material through the mesh. As a result, local mesh refinement in the area of interest, i.e. the contact zone, is possible and the mesh of the virtual tyre model of the previous work [1] can be used here.

The steady-state braking analysis is started from an inflated tyre that is mounted on a rigid rim and pressed against a rigid, smooth surface at a selected normal load. The rolling condition of the virtual tyre is set up and controlled by specifying a rotational speed and a travelling ground velocity for the tyre-wheel assembly. Consequently, a braking simulation is obtained when the product of the rolling radius $\left(r_{e}\right)$ and the angular velocity of the tyre $(\Omega)$ is smaller than the longitudinal travelling ground speed $\left(V_{X}\right)$. Under this condition a negative longitudinal wheel slip arises $(\kappa)$, which is given by [6]:

$$
\kappa=-\frac{V_{X}-\Omega r_{e}}{V_{X}}
$$

where: $r_{e}$ : rolling radius (see equation (2))

$\Omega$ : angular velocity of the tyre

$V_{X}$ : longitudinal velocity of the tyre 
The zero slip condition is reached when no longitudinal force is generated by the tyre. Also, under this free-rolling condition, the rolling radius of the tyre can be calculated from:

$$
r_{e}=\frac{V_{X}}{\Omega_{0}}
$$

where: $\Omega_{0}$ : angular velocity of the free-rolling tyre

\subsection{Model boundaries}

The FE model comprises two contact boundaries: first, the interface between the tyre and the wheel rim, and, second, the interface between the tyre tread and the ground surface. The first boundary remains unchanged from the previous work [1], that is, a constant friction coefficient of 0.5 is used between the tyre and the rim surfaces. This friction coefficient value was found to be suitable for the rolling analyses as only localised sliding between the two surfaces (which does not affect the results obtained) was found to occur. The second contact boundary is altered; instead of a constant friction coefficient an extended friction formulation is employed to capture the fundamental tread-road interaction mechanisms within the contact patch of a rolling tyre. Rubber friction varies with compound, road surface properties, temperature, contact pressure and sliding velocity. The first three of these five factors are considered to be constant and, thus, are excluded from this study. In particular, the rubber compound is assumed to maintain its properties during the brake manoeuvre (i.e., the compound does not age) and the road is treated as a continuous, rigid and smooth track surface. Temperature is disregarded as recent research suggests that the particular studied simulation results (see section 4) obtained from an ideal brake manoeuvre conducted under constant and repeatable steady-state conditions are not influenced by thermal variations. Specifically, the work by Durand-Gasselin et al. [7] shows that the thermal dependency of tyres is related to the temperature of the entire tread and the entire carcass, rather than local temperature effects. In other words, when the temperature varies through usage, the carcass stiffness properties and the contact patch friction properties change. However, the 
relationship between the carcass and the tread rubber - the characteristics which are of interest here - do not change. Hence, a friction formulation that considers separate pressure and velocity influences is used here [8], i.e.:

$$
\mu\left(p_{z}, V_{g}\right)=\mu\left(p_{z}\right) \mu\left(V_{g}\right)
$$

The influence of normal pressure is included by a pressure-dependent friction coefficient term, $\mu\left(p_{z}\right)$, which decreases to a minimum value with increasing pressure. The corresponding equation reads:

$$
\mu\left(p_{z}\right)=d_{1}\left(d_{2}+d_{3} \exp \left(d_{4} p_{z}\right)\right)
$$

where: $p_{z}$ : contact pressure

$d_{1}$ to $d_{4}$ : empirical constants

The velocity influence on the friction coefficient is considered by the second term, $\mu\left(V_{g}\right)$, which reduces to a minimum value with rising sliding speed. It is given by:

$$
\mu\left(V_{g}\right)=d_{5} \exp \left(-d_{6} V_{g}^{2}\right)+\left(1-d_{5}\right)
$$

with $\quad V_{g}$ : sliding velocity

$d_{5}$ and $d_{6}$ : empirical constants

The friction model (equation (3)) is implemented with the generalised contact model built into Abaqus. To avoid friction-related convergence difficulties, Abaqus/Standard approximates the condition of stick by permitting small relative sliding between the tread and the ground surface before the shear stresses at the interface reach the maximum available frictional shear stresses, $\tau_{c r i t}$, which are given by the product of the friction coefficient, $\mu$, (calculated with equation (3)) and the prevailing contact pressure, $p_{z}$, (see Figure 3).

Thus, for the condition of no relative motion, a "sticking" friction is introduced which approximates the shear stresses created at the tyre-road interface by:

$$
\tau_{e q}=\lambda V_{g}
$$

where $V_{g}$ is the sliding velocity and $\lambda$ is the "stick viscosity", which is given by: 


$$
\lambda=\frac{\tau_{c r i t}}{V_{g, c r i t}}
$$

where: $\quad \tau_{c r i t}=\mu p_{z}$ and $V_{g, \text { crit }}$ is the maximum allowable viscous sliding velocity.

The allowable viscous sliding velocity $V_{g, \text { crit }}$, in turn, is defined as:

$$
V_{g, c r i t}=F_{f} 2 \Omega r
$$

where $F_{f}:$ slip tolerance; $\Omega$ : angular velocity of the tyre; $r$ : radius of the tyre

Abaqus allows control of the approximation of the stick condition by specifying the slip tolerance (STOL) value, $F_{f}$. As will be discussed below, the STOL setting has some bearing on the slip characteristics of the virtual tyre.

\section{3. $\quad$ Friction model setting}

To assess the impact of the relaxation of the stick condition on virtual test results, the longitudinal force-slip characteristics obtained from steady-state rolling analyses conducted with two different STOL values are examined. The default value of 0.005 is compared with a more relaxed value of 0.02 which corresponds to values found in the literature (e.g., $[5,9])$. Also, to investigate only the influence of the STOL setting, the friction coefficient at the tread-road interface is set at a low constant value of 1.0. The specific simulation setup for the rolling analysis is described in section 3.1.

Results from this initial study (Figure 4) show that the relaxation of the stick condition affects the slip characteristics of the virtual tyre. Owing to the nature of the numerical approximation, the influence of the STOL setting is limited to low slip conditions (0 to approximately $-12 \%$ ), when a significant part of the tyre contact patch adheres to the ground. In particular, the rolling simulations set up with the default STOL value and normal loads greater than $1500 \mathrm{~N}$ show complete convergence over a wide slip ratio range, however experience numerical difficulties at about $-2 \%$ and fail to converge for smaller brake slip ratio 
values. With the relaxed friction model setting, a solution is found for all slip conditions, but the approximation of the stick behaviour affects the slip curve characteristics. That is, the virtual tyre with the higher STOL value requires a higher slip condition to generate the same force as the model set up with the default value. As a consequence, the slip stiffness of the tyre model varies with the friction model setting, i.e., higher STOL values lead to lower tyre slip stiffnesses and vice versa. Moreover, as the tyre model is subjected to all possible brake force states (although at different slip ratio values) irrespective of the STOL setting the particular simulation results examined here (i.e., deflection behaviour of the reinforcement structure) are unaffected.

In conclusion, the relaxation of the stick condition (STOL value of 0.02) is required to run simulations successfully. The specific STOL setting has some bearing on the computed slip stiffness of the tyres, but the specific results that are of interest here are valid and merit closer investigation.

\section{Virtual testing}

The racing tyre is investigated at three different normal loads $(1500 \mathrm{~N}, 3000 \mathrm{~N}$ and $4500 \mathrm{~N})$ and two different camber angles $\left(0^{\circ}\right.$ and $\left.-3^{\circ}\right)$ for a sweep of slip ratios from $0 \%$ to $-20 \%$. All rolling analyses are conducted at a constant wheel velocity of $19.4 \mathrm{~m} / \mathrm{s}$, which corresponds to the typical speeds employed for in-door experimental tests [10]. At these low velocities inertial effects can be considered negligible and will not be simulated in this study.

\subsection{Procedure}

Starting with a tyre model that is mounted on a rigid rim, inflated, cambered and loaded vertically, the steady-state rolling analysis itself is broken down into two steps. In the first step, the straight-line rolling speed and the angular velocity of the wheel are increased linearly from the stationary condition to the desired steady-state rolling condition. To guard 
against convergence difficulties, the pressure- and velocity-dependent friction coefficient is ramped up from zero to its final value during this step. In the second step, a sweep of slip ratios is simulated by varying the angular velocity of the tyre-wheel assembly while maintaining the longitudinal velocity constant.

\subsection{Data extraction}

For the analysis of the steady-state braking manoeuvre five types of data are extracted from the simulation results and post-processed with specially written Matlab scripts. The extracted data include:

(1) overall tyre forces - obtained from reaction output variables "RF" and "RM" built into Abaqus

(2) absolute velocity distribution (magnitude and direction) - obtained from field output variable "V" available in Abaqus

(3) normal pressure distribution within the contact patch - obtained from the Abaqus surface output variable "CPRESS"

(4) shear stress distribution (in longitudinal and lateral directions) within the contact patch - obtained from surface output variable "CSHEAR" available in Abaqus

(5) tyre deformations in the contact zone - assessed by displacement of the mesh nodes of the tread belt (Figure 5) relative to a reference state; required data extracted from deformed nodal coordinates which are obtained from the Abaqus field output variable "COORD"

\section{Results obtained}

Results of the longitudinal force against slip ratio characteristics, the contact pressure distribution, the shear stress distributions in the contact region and the carcass (tread belt) deflection are presented and discussed below. Data on the velocity distribution are employed to investigate rolling radius effects within the contact patch as discussed further below.

\subsection{Slip characteristics}

The longitudinal force $\left(F_{x}\right)$ against slip ratio curves for both upright and cambered tyres

(Figure 6) exhibit the expected characteristics. At low slip values a nearly linear relationship between $F_{x}$ and slip ratio exists. With rising brake slip, a peak longitudinal force is reached and a further increase of slip ratio causes a reduction of $F_{x}$ due to the 
sliding-velocity-dependent friction coefficient term (see equation (5)). Also, as expected, the magnitude of the longitudinal force generated by the tyre rises with increasing normal load.

\subsection{Contact pressure distribution}

The contact pressure distribution of the braked tyre model agrees well with the characteristics found in published data (e.g., [11]). As indicated by the average contact pressure distribution along the centre rib of the virtual tyre (Figure 7), the entire contact patch moves in the direction of the braking force, i.e., towards the rear (right), with increasing brake slip of up to $-15 \%$. Higher slip conditions lead to a drop of the generated brake force (see section 4.1) and, hence, a reduction of the longitudinal displacement of the contact zone. The longitudinal shift is accompanied by the formation of a high pressure region at the leading edge of the contact patch, which is most pronounced at slip ratios greater than $-10 \%$.

\subsection{Shear stresses and tyre deformation characteristics}

The consistency of the simulation results observed for the slip characteristics and the contact pressure distribution is also seen in the shear stress distribution. In particular, the distribution of the average longitudinal shear stress of the centre rib along the contact length at different slip ratio values (Figure 8) agrees well with published results (e.g., [12]). Beginning at the leading edge of the contact patch, the shear stresses progressively increase with contact length, indicating an adhesion region. At some point in the contact zone (the exact position depends on the prevailing slip ratio) the shear stresses reach the local value of the maximum available frictional shear stress. Then, the tread rubber loses grip and starts to slide until leaving the interface, forming a sliding zone. Accordingly, the shear stresses reduce. Also, as will be explained further below, the positive shear stresses generated at zero slip imply a variation of the local rolling radius within the contact region of the tyre.

Observation of the shear stress distribution throughout the entire contact patch of the free rolling tyre (Figure 9a) reveals two particular shear stress characteristics which are 
indicative of the deformation behaviour of the tyre structure: first, lateral contact patch shrinkage (or waisting) and, second, ply-steer.

Lateral shrinkage of the contact region implies shear forces, which oppose the contraction due to friction at the tread-road interface. This behaviour is reflected by the shear stress vectors pointing laterally outwards (to the sides of the contact patch). As reported in the previous work [1], contact patch waisting arises from the relaxation of the inflation-induced pre-strains of the tyre cords in the contact region. This narrowing effect can be visualised by plotting the lateral displacement of the nodal coordinates of the tread belt caused by increasing the vertical load on a tyre rolling on a frictionless surface by $3000 \mathrm{~N}$ (with respect to the inflated, unloaded tyre). Thus, as shown by Figure 9b, the lateral curvature of the circumferential tread belt varies across the width of the tyre - from a high curvature at the sides to zero curvature at the centre. Also, as waisting in the contact region leads to useless sliding of rubber against the road surface, the rolling resistance of the tyre increases and energy is consumed. This waste of energy can be reduced by making the tread band stiff and the sidewalls flexible.

Ply-steer generates lateral shear stresses within the entire contact patch region that point in the same direction and, thus, produce an overall side force. As the shear stresses caused by ply-steer and waisting augment each other in one half of the contact patch and oppose each other in the other half, the overall shear stress distribution at the tread-road interface of the free rolling tyre becomes asymmetric about the wheel centre plane (Figure 9a). That is, the shear stresses on the right side of the contact patch (negative y-axis) are greater than on the left side (positive y-axis). The presence of ply-steer is indicated by a slight rotation of the entire belt about the vertical axis, as shown by Figure $9 \mathrm{~b}$.

In addition to these two effects, a 'whirl' of shear stress vectors near the trailing edge of the outermost tread ribs can be observed (Figure 9a). This pattern results from local belt 
deformations, which can be ascribed to the gradual 'release' of the contact condition between the tread rubber and the road surface near the trailing edge.

At a slip ratio of $-10 \%$, the direction of the shear stresses is dominated by the generation of the brake force (Figure 10a). Only a small inclination of the shear stress vectors (primarily in the outermost tread rib) with respect to the x-axis can be observed. In the leading half of the contact patch, the shear stress vectors are inclined away from the wheel centre plane, whereas the stress vectors in the trailing half are pointing slightly towards the centre of the wheel. This shear stress distribution implies a 'rotation' of the tread belt towards the wheel centre plane. The particular belt deformation due to $F_{x}$ is shown in Figure 10b, which depicts the lateral displacement of the nodes of the tread belt mesh of a braked tyre with respect to the free rolling condition. Hence, the rotational motion of the tread belt rows is characterised by a lateral contraction in the leading half and a lateral expansion in the trailing half of the contact patch. This behaviour can be attributed to a combined effect of contact patch waisting and contact patch stretching induced by the frictional shear stresses [11]. In addition, the contact patch as a whole is displaced relative to the condition of the free-rolling tyre. As noted above, a longitudinal shift occurs due to the generation of the brake force. The longitudinal displacement is accompanied by a small lateral shift which can be ascribed to the lateral force induced by ply-steer.

In conclusion, the tread belt deformation due to $F_{x}$ may be interpreted as a combination of two motions: first, a translational displacement and, second, a rotational displacement of the belt rows.

When the tyre is inclined at a camber angle of $-3^{\circ}$, the shape of the tyre-road interface becomes trapezoidal. With this setup, the shear stress distribution at zero slip rolling (Figure 11 a) indicates the influence of three superimposed shear stress characteristics, which are induced by the deformation behaviour of the reinforcement structure of the tyre. These are: 
ply-steer and contact patch waisting (as identified with the upright tyre), and belt deformations caused by the inclination of the tyre.

As mentioned above, ply-steer influences the whole contact region. With the cambered configuration, the effect of ply-steer is best examined by the shear stress vectors of the centre rib, which point in the direction of the negative y-axis. The influence and the behaviour of belt deflections caused by normal load (waisting) and camber angle can be observed by the difference in the shear stress fields on the laden and unladen sides of the contact patch. On the unladen side, the shear stresses are rather small and point in the direction of the negative $y$-axis, whereas on the laden side, the shear stresses are significantly greater and point in the direction of tyre inclination. This stress distribution implies that the two deflection mechanisms oppose each other on the unladen side and augment each other on the laden side.

The camber induced carcass deflection characteristics are directly examined by the lateral deflection of the circumferential tread belt of the inclined tyre relative to an upright tyre (Figure $11 \mathrm{~b}$ ). To avoid frictional influences arising from tyre width effects (as will be explained later), the lateral displacement of the tread belt caused by the camber angle is investigated for a tyre rolling on a friction free surface. Hence, as shown by Figure $11 \mathrm{~b}$, inclining the tyre causes two 'types' of deflection of the tread belt. First, lateral bending of the tread belt occurs (e.g., $[13,6]$ ) which decreases from the contact patch sides towards the wheel centre plane. Second, the whole tread belt is displaced laterally in the direction of the negative y-axis.

In addition to the three factors described above, the considerable width of the virtual tyre affects the shear stress distribution. The influence can be investigated from the shear stresses within the contact patch of a cambered tyre at zero longitudinal slip (Figure 11a). Although the tyre is rolling freely, the shear stresses in ribs 1 to 4 are slightly inclined towards the front and, thus, are generating a driving force. In contrast, the shear stresses in 
the outermost rib on the laden side (rib 5) point slightly towards the rear, and correspond to a braking force. As a consequence, the rolling radius is not constant across the width of the tyre and, thus, a local rolling radius $[6,12]$ may be defined. Based on the definition of the rolling radius (equation (2)), the local rolling radius at any location in the tyre contact patch will be described as the ratio of the forward velocity of the tread surface at the particular coordinate and the wheel speed for the condition when no longitudinal force is generated. In general, this ratio can be expressed as:

$$
r_{e i}=\frac{V_{x}-V_{x i}}{\Omega}
$$

where $V_{x}$ : longitudinal velocity of the wheel centre

$V_{x i}$ : local longitudinal velocity of the tread within the contact patch

$\Omega$ : angular velocity of the wheel

As a tyre can only produce forces on friction surfaces, the local rolling radius within the whole contact patch can be easily obtained from FE rolling simulations on a frictionless surface. Results from this type of simulations are shown in Figure 12.

As expected, FE simulations with a cambered tyre (Figure $12 \mathrm{~b}$ ) reveal that the rolling radius is greater on the unladen side than on the laden side. However, owing to the complex tyre deformations and changing longitudinal and lateral contact patch dimensions of the inclined tyre, the variation of the rolling radius is not symmetric about the wheel centre plane, rather about the centroid of the contact patch.

Plotting the longitudinal forces generated by the individual tread ribs against slip ratio (Figure 13) highlights this tyre-width effect once more. At zero slip, rib 5 generates a braking force that is balanced by the driving forces produced by the other ribs. At around $-1 \%$ slip, the driving forces vanish and all tread ribs start to generate brake forces. Also, the individual contribution of the tread ribs to the overall tyre force corresponds to the uneven distribution of the contacting area. 
Similar to the upright tyre, the introduction of brake slip with the cambered tyre generates shear stress vectors that are primarily pointing towards the rear (Figure 14a). Moreover, the shear stress field shows a comparable pattern to the one observed for the tyre at zero camber. Hence, the shear stress vectors near the leading edge of the contact patch are slightly inclined away from the wheel centre plane. In the trailing half, the shear stress vectors are directed slightly towards the centre of the wheel. Therefore, as described for the upright tyre, a rotational displacement of the tread belt rows occurs, which is accompanied by a translational displacement of the entire contact patch, as shown by Figure 14b.

\section{Conclusions}

Physically realistic rolling-tyre behaviour has been demonstrated by the FE model. The virtual tyre allows extraction of detailed results which are not easily obtainable through measurements on an actual tyre. The simulation results and experimental data from the literature show substantial agreement in respect of, a) deflection behaviour, b), slip characteristics, c), normal force and d) shear stress distributions over the contact region.

Examination of the deflection behaviour of the reinforcement structure of the rolling tyre revealed a strong interdependence between the carcass deformation characteristics and the shear stress distribution within the contact patch. In particular, four factors that influence the characteristics of the carcass and, consequently, the shear stress distribution within the contact patch were identified:

- Normal load and camber angle: carcass deformations resulting from normal loading and camber angle were found to induce lateral shear stresses which oppose each other on the unladen side and augment each other on the laden side.

- Brake force: The generation of brake force caused longitudinal displacement and stretching of the contact patch. These deflections are accompanied by lateral shrinkage (or "waisting") in the contact region. In the leading half of the contact patch, the shear stresses point towards the wheel centre plane, whereas in the trailing half the shear stresses are in the opposite direction.

- Tyre width: The longitudinal force distribution across the tyre width showed the generation of localised driving and braking forces even for free rolling conditions. This behaviour is indicative of a local rolling radius variation which can be directly measured with the FE model. 
These results aid understanding of contact patch characteristics and allow insights on

the impact of tyre design / architecture on the behaviour of a rolling tyre. Also, the

information obtained here helps in the creation of dedicated tyre models as shown in the second part of this two-part paper [14].

\section{References}

[1] Gruber, P., Sharp, R. S. and Crocombe, A. D., Friction and camber influences on the static stiffness properties of a racing tyre, Proc. IMechE 222, Part D: J. Automobile Engineering (2008), pp. 1965-1976.

[2] Trinko, M. J., Tire Stress Analysis, in The Pneumatic Tire, J.D. Walter and A.N. Gent, National Highway Traffic Safety Administration U. S. Department of Transportation, Washington DC, 2005, pp. 206-229.

[3] Rao, K. V. N., Kumar, R. K., Mukhopadhyay, R., Misra, V.K., A study of the relationship between Magic Formula coefficients and tyre design attributes through finite element analysis, Vehicle Syst. Dyn. 44(1) (2006), pp. 33-63.

[4] International Organization for Standardization (ISO), ISO 8855:1991 Road vehicles Vehicle dynamics and road-holding ability - Vocabulary (1991), Geneva.

[5] Abaqus version 6.7 documentation, Dassault Systèmes, Simulia, Providence, Rhode Island, USA, 2007.

[6] Pacejka, H. B., Tyre and vehicle dynamics, $2^{\text {nd }}$ Edition, Butterworth-Heinemann, Oxford, 2006.

[7] Durand-Gasselin, B., Dailliez, T., Moessner-Beigel, M., Knorr, S., Rauh, J., Assessing the Thermo-mechanical TameTire Model in Off-line Vehicle Simulation and Driving Simulator Tests, IAVSD 2009, Stockholm, 2009.

[8] Savkoor, A. R., Models of Friction, in Handbook of Materials Behavior Models, Volume 2 Failures of Materials, J. Lemaitre, Academic Press, London, 2001, pp. 700-759.

[9] Qi, J., Herron, J. R., Sansalone, K. H., Mars, W. V., Du, Z. Z., Snyman, M. and Surendranath, H., Validation of a Steady-State Transport Analysis for Rolling Treaded Tires, Tire Sci. Technol. 35(3) (2007), pp. 183-208.

[10] Arosio, D., Braghin, F., Cheli, F., Sabbioni, E., Identification of Pacejka's scaling factors from full-scale experimental tests, Vehicle Syst. Dyn. Suppl. 43 (2005), pp. 457-474.

[11] Clark, S. K., Mechanics of Pneumatic Tires, $2^{\text {nd }}$ edition, US Dept. of Transportation, National Highway Traffic Safety Administration, Washington, D.C, 1981.

[12] Pottinger, M. G., Contact Patch (Footprint) Phenomena, in The Pneumatic Tire, J.D. Walter and A.N. Gent, National Highway Traffic Safety Administration U. S. Department of Transportation, Washington DC, 2005, pp. 231-285.

[13] Fiala, E., Seitenkräfte am rollenden Luftreifen, VDI Zeitschrift 96(29) (1954), pp. 973979.

[14] P. Gruber, R.S. Sharp, and A.D. Crocombe, Normal and shear forces in the contact patch of a braked racing tyre; Part 2: Development of a physical tyre model, Veh. Syst. Dyn. 50(3) (2012), pp.339-356. 

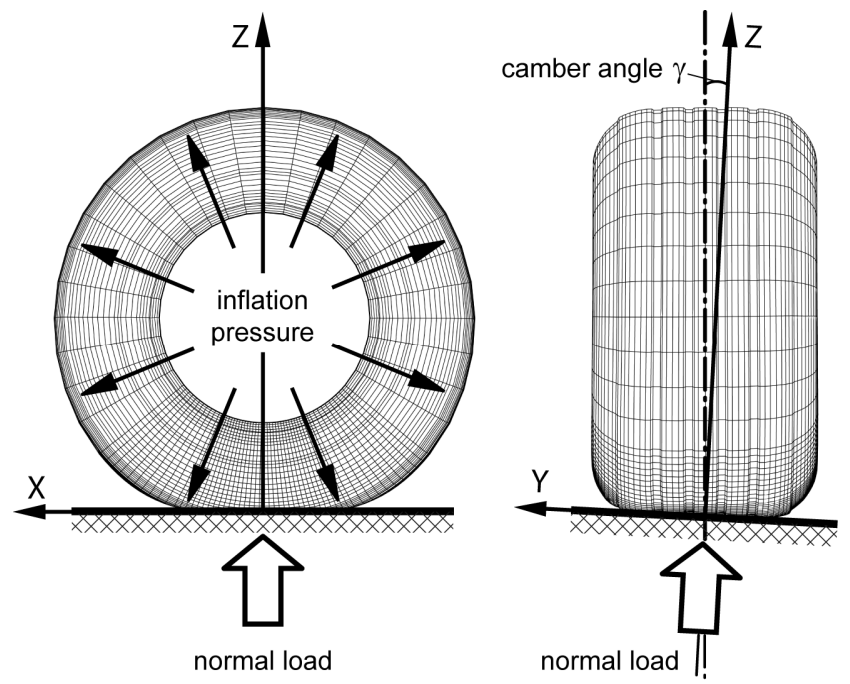

Figure 1: Validated tyre model [1].

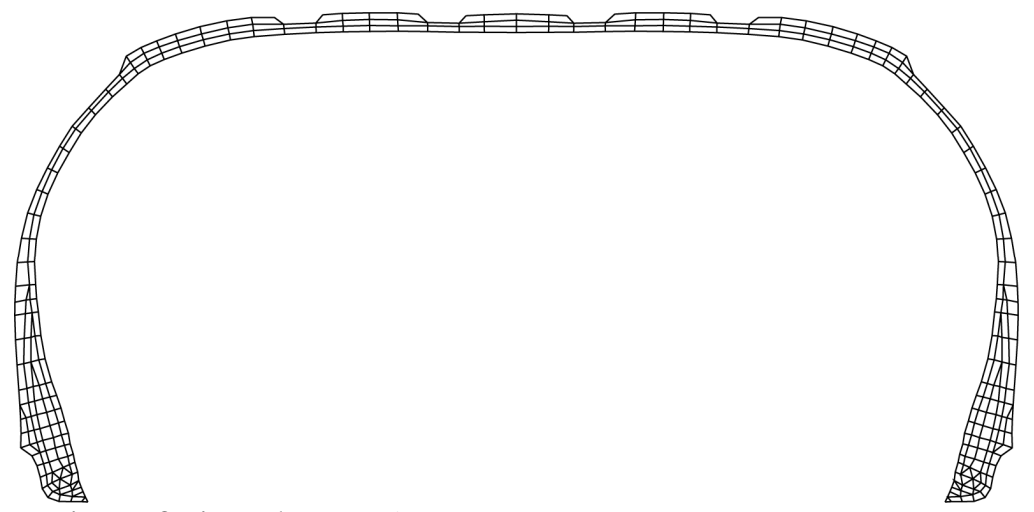

Figure 2: Cross-section of virtual tyre [1].

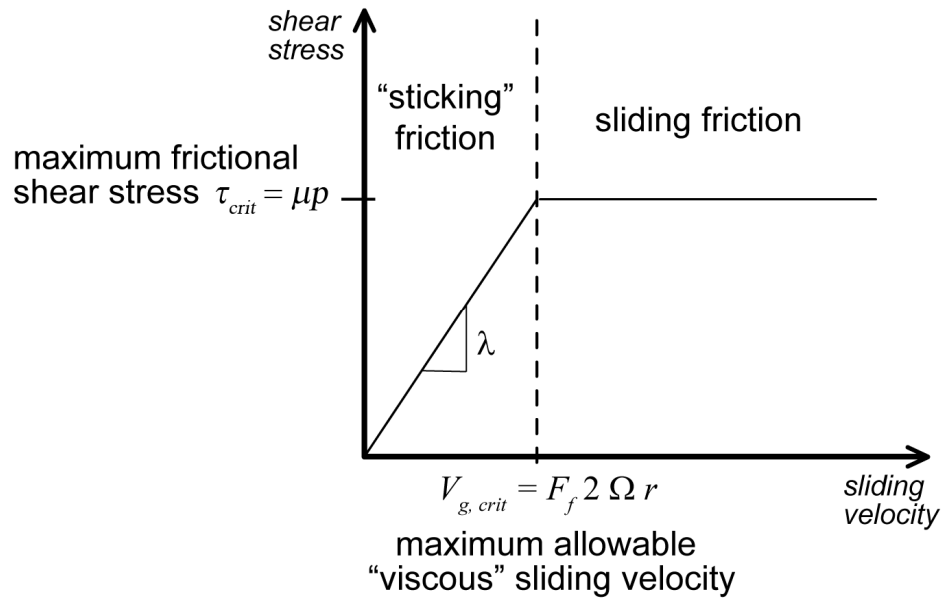

Figure 3: Schematic plot of the shear stress against sliding velocity relationship for sticking and slipping friction. 


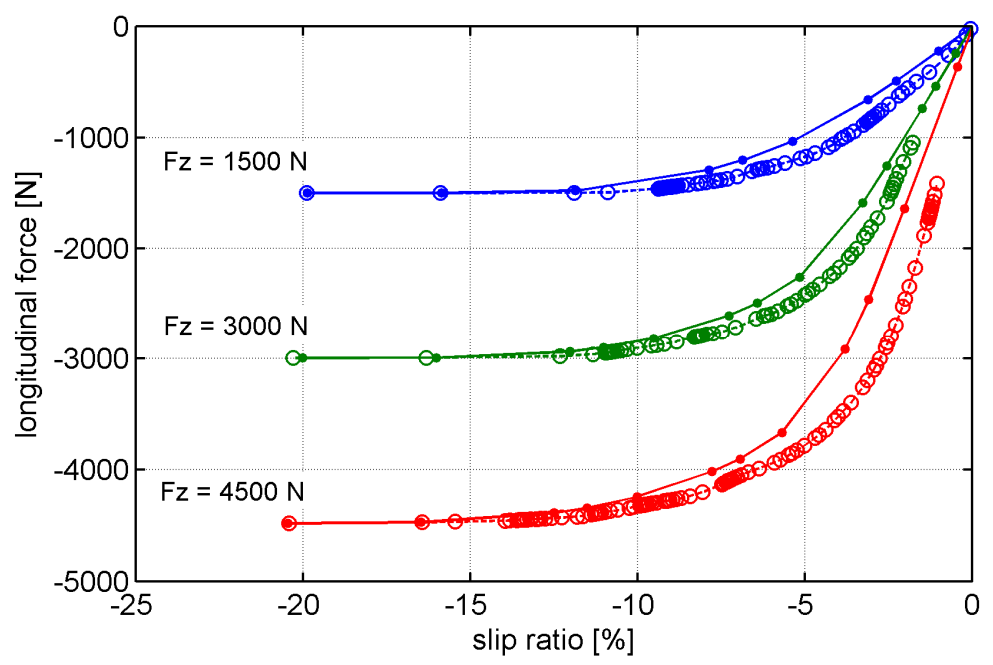

Figure 4: Longitudinal force against slip ratio of the upright tyre model with two different slip tolerance (STOL) settings. Open circles indicate the default STOL setting and solid circles mark the $\mathrm{STOL}=0.02$ setting.

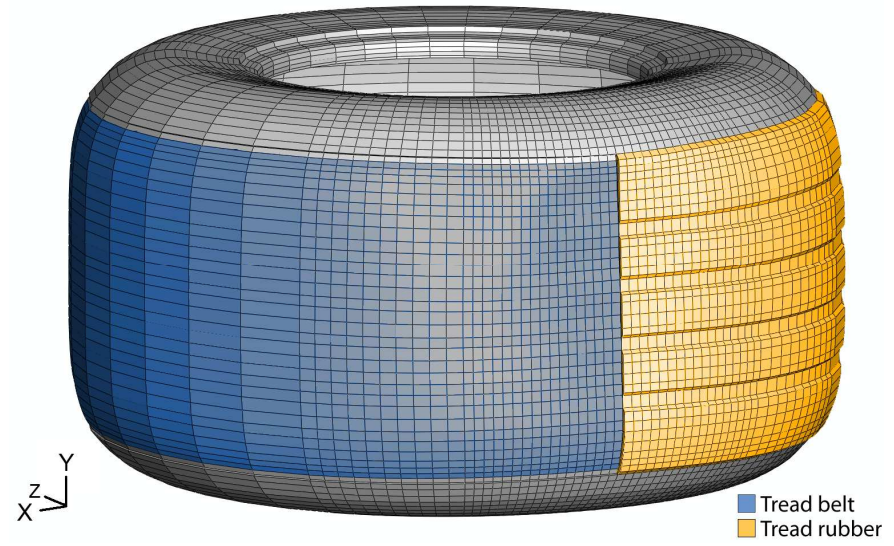

Figure 5: FE mesh of the virtual tyre model with a cutaway view of the tread belt. Deflected tyre shown from underneath.

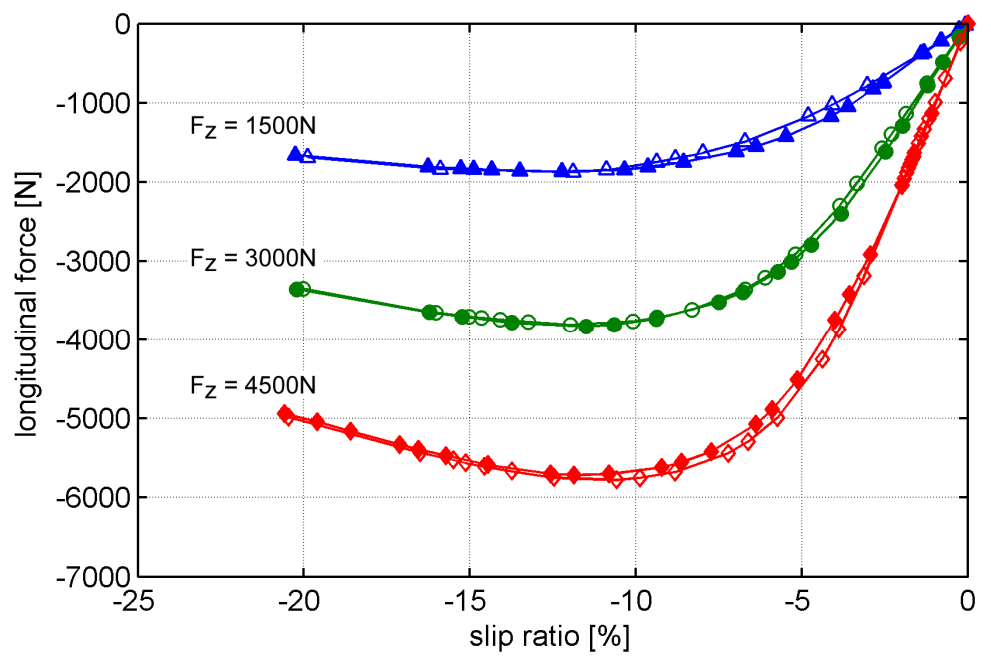

Figure 6: Longitudinal force against slip ratio at three different normal loads for an upright tyre (solid line with open markers) and cambered tyre (dashed line with solid markers). 


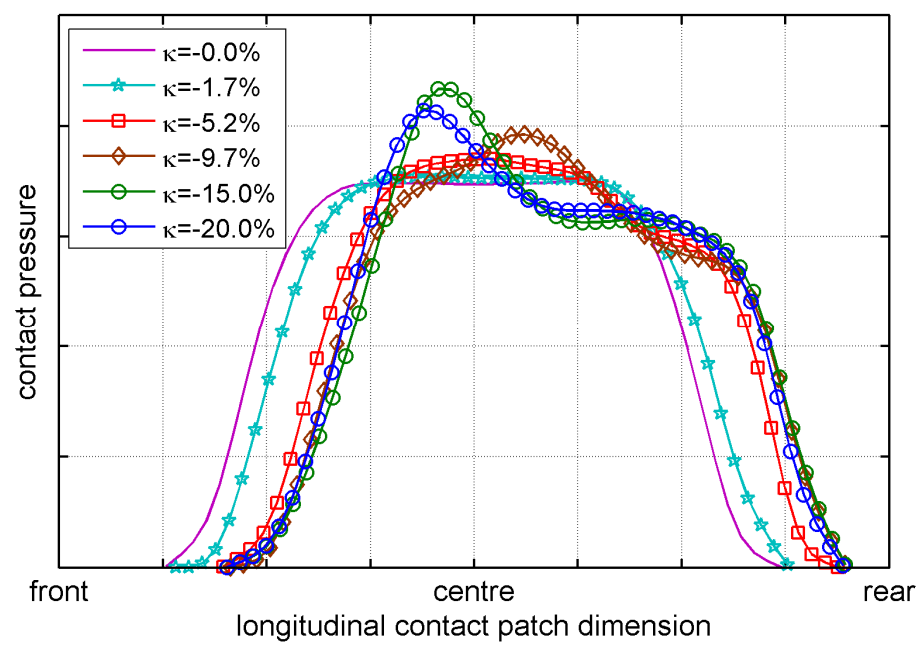

Figure 7: Average contact pressure distribution of the centre tread rib against longitudinal coordinate as a function of slip ratio (upright tyre at a normal load of $3000 \mathrm{~N}$ ).

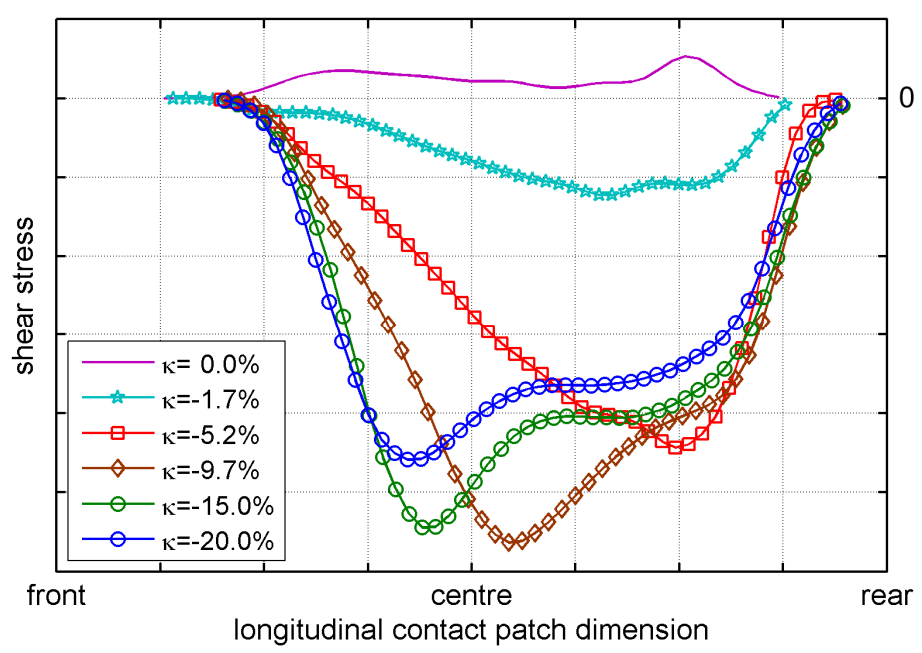

Figure 8: Average longitudinal shear stress distribution along the longitudinal direction of the centre tread rib at different slip ratios (upright tyre at a normal load of $3000 \mathrm{~N}$ ). 


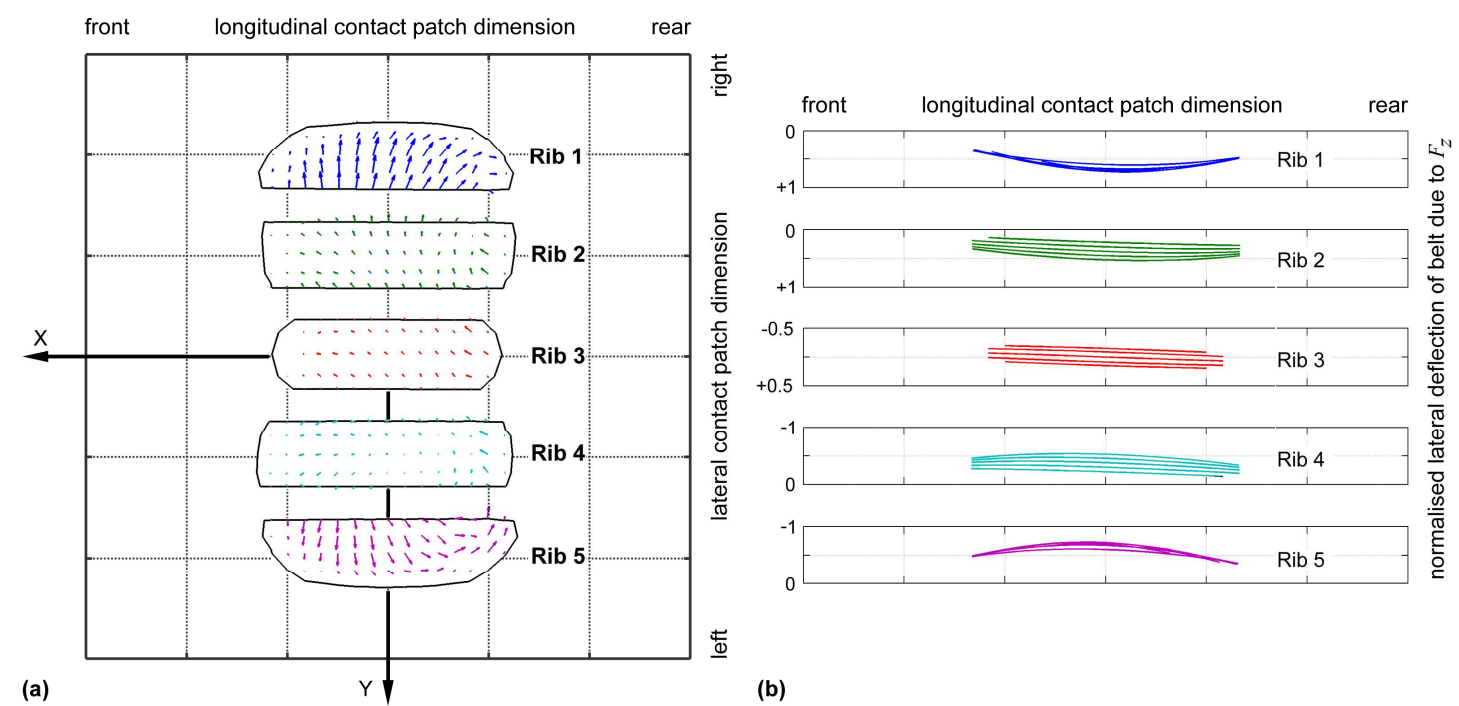

Figure 9: Upright tyre rolling at 0\% slip: (a) shear stress vector plot at a normal load of $3000 \mathrm{~N}$ and (b) normalised lateral displacement of the belt coordinates caused by normal loading of $3000 \mathrm{~N}$ on a frictionless surface with respect to an unloaded, inflated tyre. Each line represents a longitudinal line of mesh nodes.

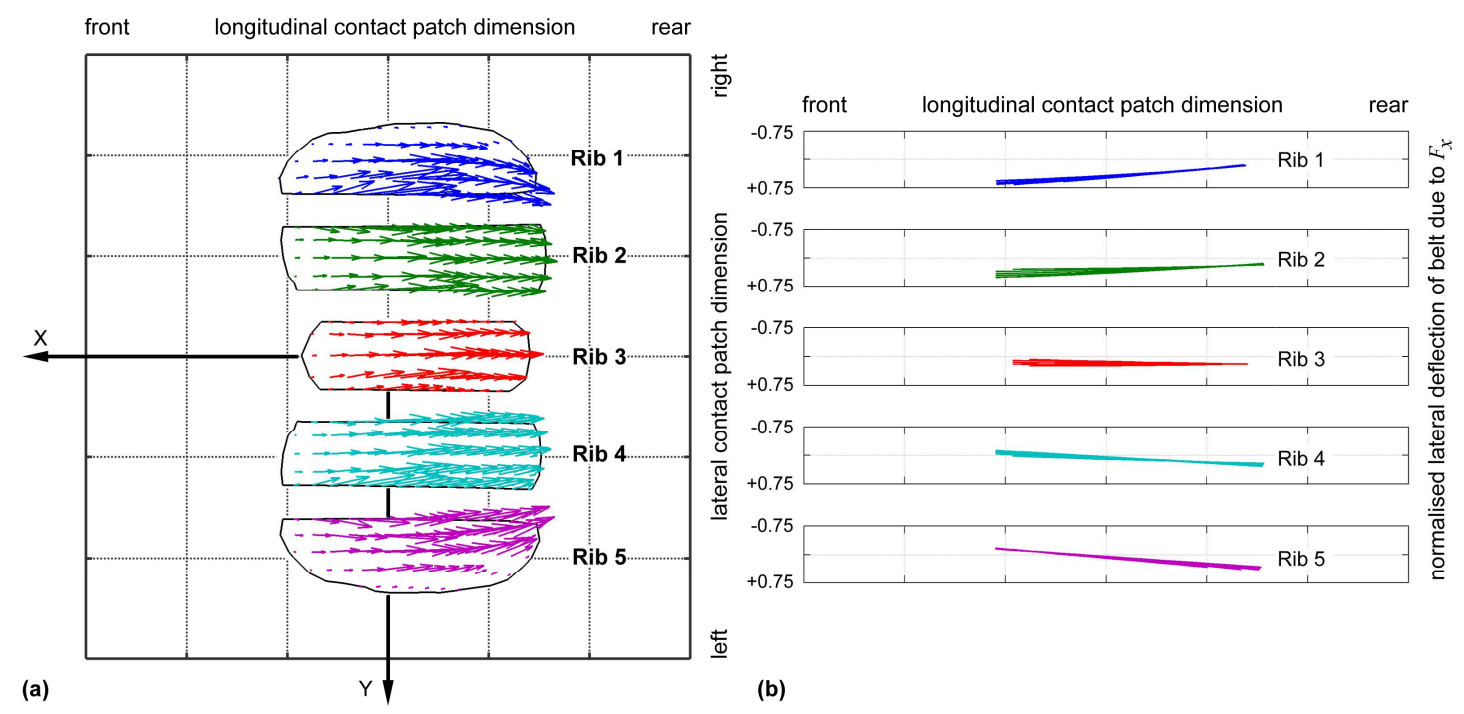

Figure 10: Upright tyre at a normal load of $3000 \mathrm{~N}$ rolling at $-10 \%$ slip: (a) shear stress vector plot and (b) normalised lateral displacement of the belt coordinates caused by braking at a slip ratio of $-10 \%$ with respect to a tyre rolling freely in a straight line. 


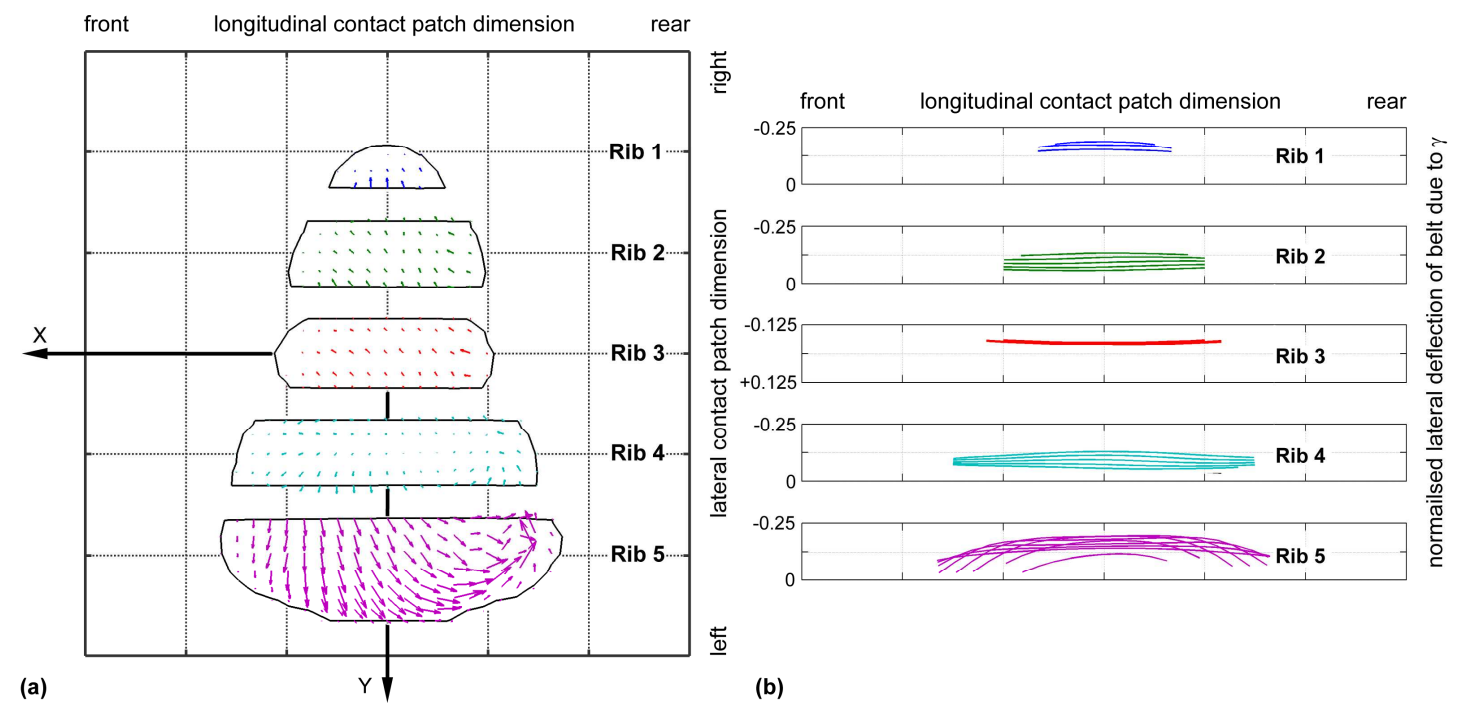

Figure 11: (a) Shear stress vector plot at 0\% slip (cambered tyre at a normal load of $3000 \mathrm{~N}$ ); (b) Normalised lateral displacement of the belt coordinates with respect to an upright tyre caused by a camber angle of $-3^{\circ}$. Tyre rolling on a frictionless surface.
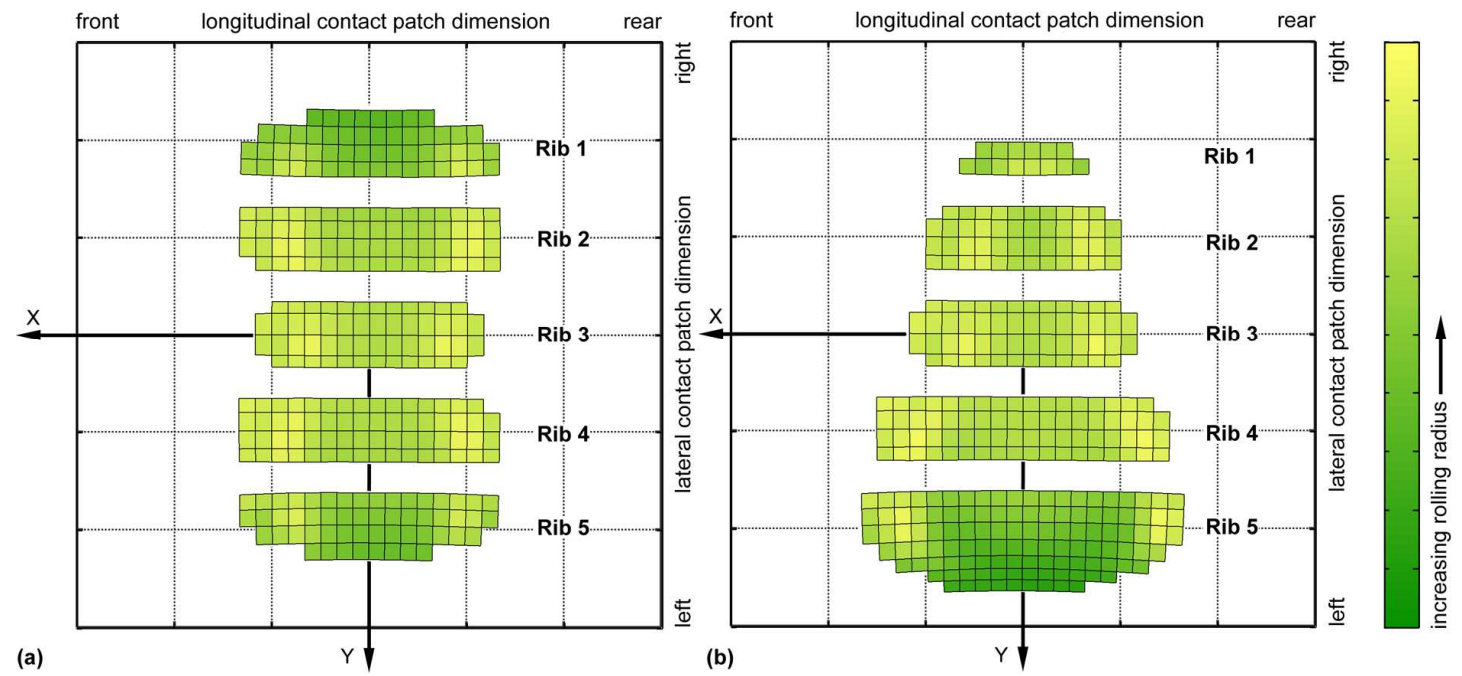

Figure 12: Rolling radius within the contact patch of (a) the upright tyre and (b) the cambered tyre $\left(\gamma=-3^{\circ}\right)$ rolling on a frictionless surface (FE simulations at a normal load of $3000 \mathrm{~N})$. 


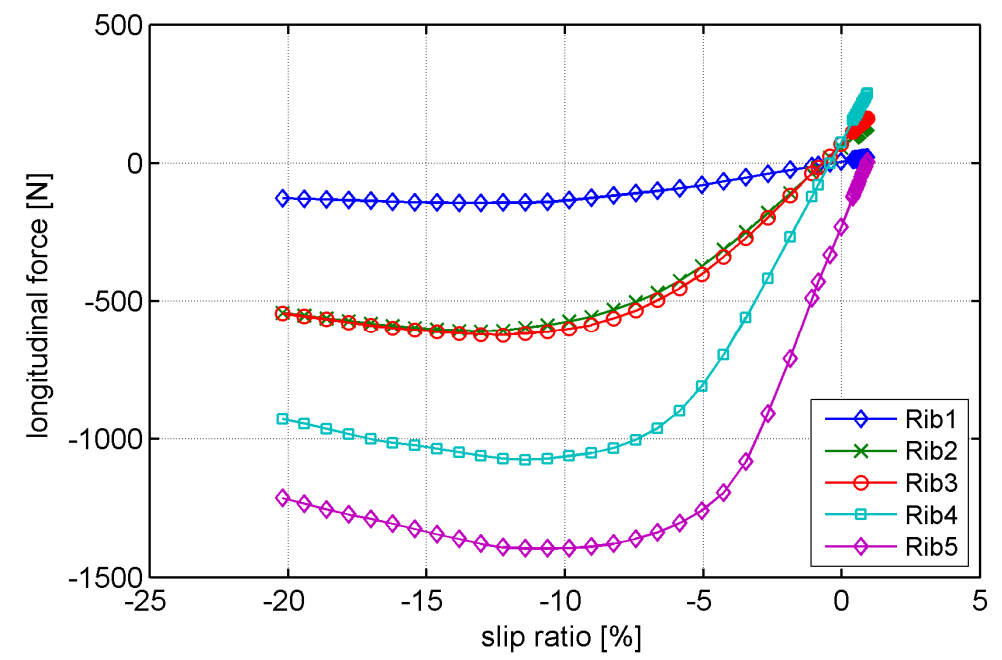

Figure 13: Longitudinal force generated by each tread rib against slip ratio at $-3^{\circ}$ camber angle and a normal load of $3000 \mathrm{~N}$.

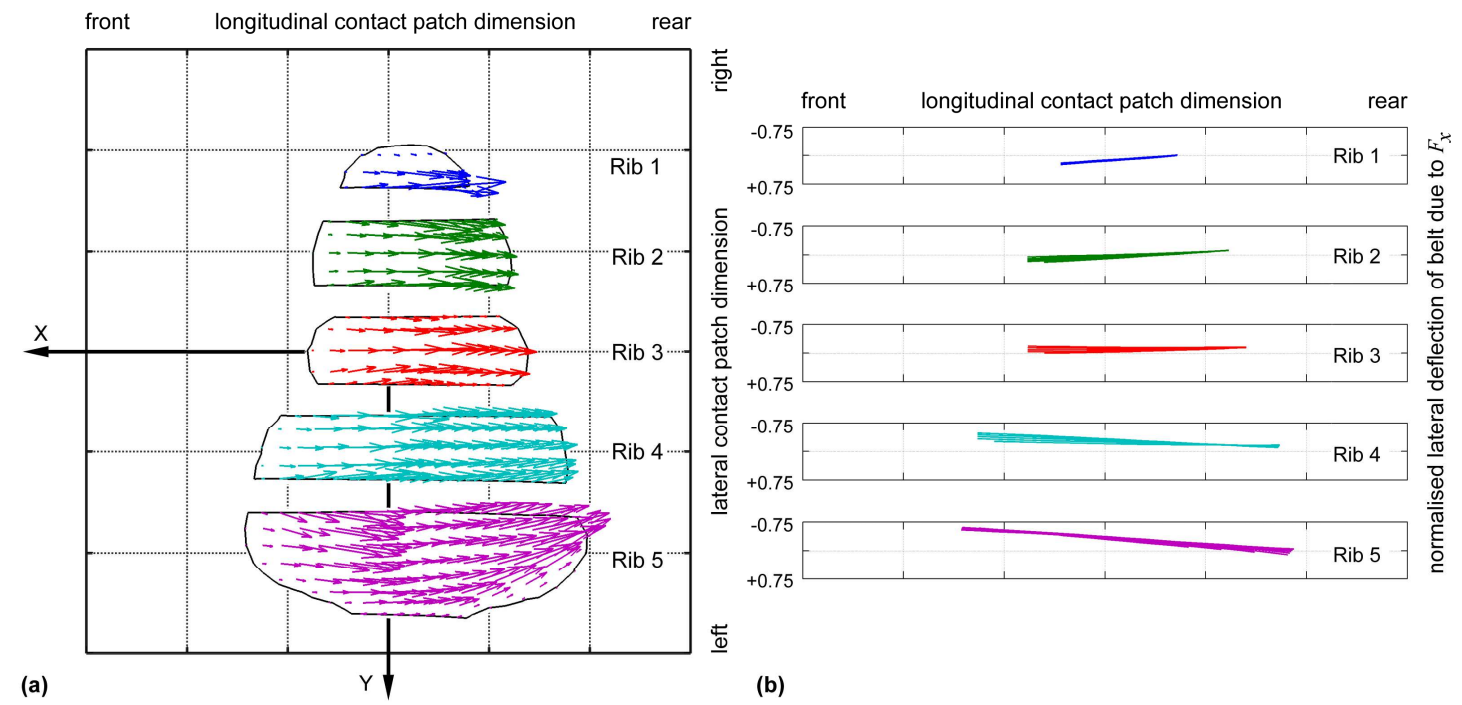

Figure 14: (a) Shear stress vector plot at $-10 \%$ slip (cambered tyre at a normal load of $3000 \mathrm{~N}$ ); (b) Normalised lateral displacement of the belt coordinates caused by braking at a slip ratio of $-10 \%$ with respect to a tyre rolling freely in a straight line. 